\title{
Poésie et didactique de la poésie dans l'enseignement du FLE en Iran
}

(Teaching)Poetry in TFFL (Teaching French as Foreign Language) in Iran

Jaleh Kahnamouipour et Akbar Abdollahi

\section{(2) OpenEdition}

\section{Journals}

Édition électronique

URL : http://journals.openedition.org/pratiques/5289

DOI : 10.4000/pratiques.5289

ISSN : 2425-2042

Éditeur

Centre de recherche sur les médiations (CREM)

Référence électronique

Jaleh Kahnamouipour et Akbar Abdollahi, « Poésie et didactique de la poésie dans l'enseignement du FLE en Iran », Pratiques [En ligne], 179-180 | 2018, mis en ligne le 31 décembre 2018, consulté le 10 décembre 2020. URL : http://journals.openedition.org/pratiques/5289; DOI : https://doi.org/10.4000/ pratiques.5289

Ce document a été généré automatiquement le 10 décembre 2020.

(c) Tous droits réservés 


\title{
Poésie et didactique de la poésie dans l'enseignement du FLE en Iran
}

\author{
(Teaching)Poetry in TFFL (Teaching French as Foreign Language) in Iran
}

Jaleh Kahnamouipour et Akbar Abdollahi

À quoi te servira un plateau de roses ? Emporte plutôt une feuille de mon Parterre de

roses.

La fleur dure seulement cinq ou six jours, et ce parterre sera toujours beau Saadi, Le Parterre de roses, XIII ${ }^{e}$ siècle

\section{Poésie et didactique du FLE en Iran}

1 Dans un entretien que l'on peut avoir avec un Occidental, l'interlocuteur recourt rarement à une citation de W. Shakespeare, de V. Hugo, ou de Novalis. Mais pour tout Iranien, la poésie fait partie de la vie quotidienne et recourir aux poètes célèbres de son pays, pour illustrer ou appuyer ce qu'il est en train de dire ou de raconter, est quelque chose d'ordinaire et de fréquent ${ }^{1}$. Il existe actuellement plusieurs conceptions de l'enseignement du français langue étrangère (FLE), conceptions fondées sur l'imitation, l'imprégnation, la répétition ou la comparaison. Pour les raisons que nous allons présenter plus loin, nous proposons, dans le cadre de l'enseignement de la poésie dans les cours du FLE en Iran, une approche fondée sur la comparaison. Cette approche prend appui sur le « déjà-là » (Marin \& Crinon, 2017) des apprenants. D'après M.-F. Carnus et A. Terrisse (2013), le «déjà-là » désigne les filtres hérités de l'expérience passée, qui modifient et biaisent les objectifs préconçus de l'enseignement (Vergnaud, 2013). La question qu'on peut se poser est de savoir si le « déjà-là » des apprenants iraniens est un obstacle ou un levier pour entrer dans la poésie française et la langue française. Dans cet article notre première hypothèse est la suivante : le déjà-là des œuvres persanes serait un levier fort intéressant, contredisant ce qu'on appelait naguère le bain linguistique intégral, sans rapport avec la langue-culture d'origine. 
2 Dans notre pays, l'interaction entre l'apprenant-lecteur et le texte poétique peut donc être considérée comme une expérience singulière dans l'apprentissage d'une langue vivante. La lecture du texte poétique est une activité familière pour l'apprenant-lecteur iranien qui vit dans un pays où tous les ans, parmi les publications best-sellers, on voit la republication du Divan de Hafez, de nombreux extraits de Masnavi de Mowlânâ Rûmî et de La lettre des Rois de Ferdowsi. En effet les étudiants iraniens, surtout ceux qui préparent un diplôme universitaire en langue étrangère - française ou autre -, sont immergés dans une culture poétique nationale très riche et même si on déplore de nos jours le peu d'intérêt des plus jeunes pour la littérature en général, et pour la poésie en particulier, notre expérience de l'enseignement du FLE dans les universités iraniennes montre qu'il existe toujours dans chaque classe de FLE, des étudiants s'étant déjà exercés à faire des vers.

3 Il est aussi à noter que l'apprenant iranien appartient à un peuple pour qui la tombe de ses grands poètes est un lieu sacré aussi précieux qu'un mausolée. Dans ce contexte, on pourrait dire que les tombeaux de Chiraz (Hafiz, Saadi), d'Hamadān (Baba Taher) et de Meshed (Ferdowsi) sont, d'une certaine façon, des poèmes ou l'image des œuvres complètes des poètes dans l'imaginaire collectif des Iraniens. La visite du tombeau accompagnée des lectures, des rites, des exorcismes, des vœux et des prédictions serait elle-même une démarche poétique : une sorte de visite au "paradis", mot qui vient de l'ancien persan signifiant jardin. Dans cette perspective, l'une des conceptions de la poésie est celle du tombeau ; la poésie, et la littérature en général seraient un rapport aux morts.

Cette conception de la poésie comme tombeau (cf. en France, S. Mallarmé et les musiciens comme C. Debussy et M. Ravel : Le Tombeau de Couperin), mais aussi comme jardin-Eden et donc comme lieu de pèlerinage, et donc le pèlerinage comme activité de poésie constituent une particularité importante de l'enseignement/apprentissage de la poésie dans le contexte iranien. On pourrait dire que le voyage à Paris pour un Iranien, sur les traces de C. Baudelaire ou des Misérables de V. Hugo, en serait un équivalent. Il s'agit en général de la construction d'un rituel, d'une sacralisation, ici fondés sur deux imaginaires collectifs : iranien de la poésie et iranien de la France, ou plutôt le stéréotype de la France-Paris-Culture pour un Iranien.

5 L'hypothèse secondaire que nous proposons ici porte sur les dimensions psycholinguistique et socioculturelle de l'enseignement de la poésie française en Iran. À partir de cette hypothèse, nous essayerons de voir de plus près le lien entre la langue et la poésie dans le contexte de l'apprentissage d'une langue vivante.

6 Nous supposons que l'apprentissage d'une langue étrangère bouleverse l'équilibre psychique de l'apprenant, le met en danger, le fait retourner en enfance (in-fans en latin, bébé qui ne parle pas encore), dans son monde imaginaire singulier. Nous supposons que la poésie par son rapport à la substance de la langue, au caractère physique, émotionnel de la langue, par ses lallations et répétitions, répare le choc de l'altérité linguistique en offrant des compensations identitaires et narcissiques, que la langue maternelle n'avait peut-être pas offertes à ce point à l'école.

7 La dimension culturelle de l'enseignement de la poésie dans les cours du FLE en Iran est aussi assez importante. D'abord à l'heure où l'interculturel est au cœur des débats politiques, sociaux, éducatifs et didactiques, pourquoi dans l'apprentissage d'une langue étrangère ne pas recourir à une étude comparative entre deux ou plusieurs textes littéraires, y compris poétiques, pour assurer une véritable compétence lectorale et pour 
conduire l'apprenant au dialogue interculturel tout en utilisant les acquis de ce dernier dans sa propre culture? Établir des passerelles entre les cultures permet d'une part d'unir l'apprentissage d'une langue à la connaissance de la culture de cette langue, et d'autre part, la culture étrangère à la culture maternelle.

En ce qui concerne la fonction culturelle du texte poétique, il faut dire que ce dernier véhicule des visions du monde : celle du poète (imaginaire singulier) ou celles du groupe social auquel appartient le poète (imaginaires collectifs²). Sur le plan social aussi on peut se demander si ces imaginaires sont des obstacles ou des leviers pour entrer dans la poésie et la langue de l'autre. Dans ce cadre nous faisons l'hypothèse que la poésie permettrait également de sensibiliser l'apprenant à une autre culture que la sienne et à une "autre façon d'être au monde", ce qui lui permettrait de découvrir d'autres conceptions du monde, d'autres modes de vie et de pensée. Par le biais d'une étude comparative entre un texte poétique en français et un autre appartenant à la propre langue de l'apprenant, partageant le même thème, l'apprenant serait amené, par comparaison, à relativiser sa propre culture, à prendre conscience que toute culture a droit au respect. Il s'agit ici d'une finalité éthique pour l'enseignement de la poésie française dans les cours du FLE.

\section{Didactique de la poésie : un champ à construire}

Les discours sur la poésie et la didactique de la poésie dans l'enseignement du FLE en Iran mêlent généralement des niveaux différents d'analyse. Pour une exploitation pertinente des textes poétiques en classe de français, on a intérêt à bien cerner les plans psychoaffectif (imaginaire singulier des apprenants), socio-culturel (imaginaire collectif des apprenants), linguistique et probablement métalinguistique, voire méta-poétique. Dans cette perspective, avant de présenter le corpus de notre recherche, il ne sera pas inutile de préciser les contextes sociocognitif et psycho-affectif de la poésie et de la didactique de la poésie en Iran.

\section{Dimension sociocognitive de la poésie en Iran}

Dans le contexte de l'enseignement/apprentissage de la langue française en Iran, la culture poétique nationale des apprenants peut être envisagée selon deux aspects différents : les étudiants iraniens sont immergés dans une culture poétique nationale très riche mais cette riche tradition poétique iranienne se présente parfois comme un obstacle à l'appréhension de la poésie étrangère. Cet ancrage dans la poésie iranienne, représentant pour chaque apprenant son intime personnel et national, l'empêche-t-il de s'ouvrir à la langue et à l'imaginaire collectif des autres, ou au contraire en facilite-t-il l'accès, mais à quelles conditions de didactique et d'ergonomie? Nous allons discuter ce point plus loin dans l'analyse des discours des étudiants iraniens.

\section{Dimension psycho-affective de la didactique de la poésie en Iran}

11 Nous supposons qu'un processus d'apprentissage à la fois collectif et individuel fondé sur l'introduction des textes poétiques pertinents dans la classe du FLE en Iran peut remédier au traumatisme occasionné potentiellement par l'immersion dans une nouvelle langue. La nouvelle langue ramène l'apprenant à l'enfance, il le fait en quelque sorte "infans ", 
terme de S. Ferenczi, disciple de S. Freud, du latin infans qui désigne l'enfant qui n'a pas encore acquis le langage. D. Winnicott et M. Klein iraient dans le même sens. En langue étrangère celui qui ne peut pas encore parler bien, parle comme un enfant, avec plein d'erreurs et d'approximations. Sur le plan social - collectif - on a vu qu'il y avait de la mort dans la poésie, du manque à dire, de l'impossibilité de dire, mais il y a, en récompense, une renaissance, la naissance à l'altérité des autres, et de soi comme autre (Favriaud, Vinsonneau \& Poletto, 2017). Dans le choix des poèmes on peut viser ce niveau affectif, symbolique et décentrant. Rassurer et permettre un abandon correspondent également à deux gestes professionnels majeurs en pédagogie : la sécurisation et l'aide au lâcher-prise (Jorro, 2002 ; Bucheton, 2005). Dans cette perspective, les textes poétiques qui présentent des thèmes liés à un intérêt pédagogique, à savoir les textes qui peuvent parler à tout le monde et que tout le monde peut parler, peuvent «dynamiser la rencontre des mentalités et éclairer la rencontre et la découverte de soi par l'expérience de l'Autre » (Shayegan, 2017, p. 3).

Pour prendre en considération la dimension psycho-affective du texte poétique dans l'enseignement du FLE, plusieurs activités orales ou écrites s'offrent à l'apprenant. Si on fait une lecture à haute voix, il est évident qu'une dimension physique, corporelle et vocale est sollicitée; on peut faire l'expérience tous les jours que les grands chanteurs d'opéra parlent très bien les trois ou quatre langues dans lesquels ils jouent sur scène. $\mathrm{Si}$ on demande aux apprenants à quoi le poème leur fait penser, à quels souvenirs personnels il peut être relié, on accorde une importance à la dimension psycho-affective du texte poétique dans l'enseignement du FLE. Cette démarche peut constituer une phase de l'ancrage dans la subjectivité de l'apprenant. Il est intéressant de noter que cette activité d'enrôlement est issue des applications didactiques de la théorie de la réception développée par H. R. Jauss, W. Iser, U. Eco, P. Bayard, M. Picard, etc. Ce genre d'activités se distingue de la démarche traditionnelle qui demande aux apprenants de relever les thèmes préconçus d'un texte littéraire. La question est donc de savoir quel genre d'activité on choisit eu égard aux plans didactiques visés.

Dans l'usage pédagogique du texte poétique, on peut envisager les activités comme lire le poème, étudier la versification, dégager la structure du texte poétique, transformer le poème en récit, écrire la biographie du poète ou en faire un exposé oral, traduire le poème. Dans le cas présent d'une étude comparative entre deux ou plusieurs poèmes en langues étrangère et en langue maternelle, nombreux sont les genres d'activité à partir des textes poétiques, tels que : faire une comparaison entre les points de vue des poètes ; amener l'apprenant à la réalisation des dossiers interculturels en langue étrangère sur les thèmes traités dans les poèmes en question et faire appel aux connaissances antérieures et extralinguistiques de l'apprenant, notamment à ses acquis en langue maternelle et lui permettre de devenir un lecteur compétent en langue étrangère. Dans cette perspective, nous suivons une démarche qui commence par des activités mobilisant le sujet imaginaire ou émotionnel pour arriver aux activités plus intellectuelles prenant en compte le déjà-là des apprenants.

14 S'agissant d'une approche centrée sur l'apprenant du niveau intermédiaire et avancé dans les universités iraniennes - l'apprenant qui prépare un diplôme en FLE -, la communication poétique en raison de sa richesse au niveau langagier, culturel et interculturel, permet de mobiliser les aspects, cognitif, émotif, imaginatif et créatif. Dans ce contexte on peut ainsi s'interroger sur la place des activités poétiques dans les modes 
de construction des compétences langagières, communicatives et culturelles en langue étrangère.

\section{Description et analyse du corpus}

Afin d'étudier «les connaissances reliées à un imaginaire collectif» et «les traces de l'imaginaire singulier » dans les discours des étudiants sur la poésie et sur la didactique de la poésie dans le contexte iranien, nous avons utilisé le concept de représentations sociales. Le recueil des données a été effectué auprès de deux groupes d'étudiants en licence et en master à l'université de Téhéran. Il s'agit d'une étude qualitative par entretien semi-dirigé. Nous ne présentons ici que les résultats de l'analyse de contenu de deux entretiens semi-dirigés, individuels et approfondis (Annexe 1) avec les étudiants de langue et littérature françaises effectués en l'année universitaire 2017-2018. Ce que nous voudrions montrer ici plus particulièrement c'est comment l'expérience de l'apprentissage de la poésie française peut être vécue et pensée par les étudiants iraniens.

La notion de représentation mobilisée dans cette recherche constitue un système de connaissances partagées collectivement qui est influencé par les circonstances sociales et historiques. S. Moscovici (1984, p. 10-11) définit la représentation sociale comme « un système de valeurs, de notions et de pratiques ayant une double vocation. Tout d'abord, d'instaurer un ordre qui donne aux individus la possibilité de s'orienter dans l'environnement social, matériel et de le dominer, ensuite, d'assurer la communication ». Ces représentations jouent donc un rôle constructif dans l'interprétation du monde et dans l'élaboration de la conduite et l'échange. Quant à leur importance pour l'enseignement des langues vivantes, nous savons qu'« Elles ouvrent à la fois un champ de savoirs pratiques, un "horizon d'attentes", un espace d'expériences et d'action » (Moore \& Py, 2008, p. 273). En s'engageant dans l'étude des représentations sociales, plusieurs auteurs ont recours à la recherche qualitative (Legendre, 2005 ; Savoie-Zajc, 2004). La recherche qualitative est définie par L. Savoie-Zajc (2004, p. 126) comme « un courant animé du désir de comprendre le sens de la réalité des individus; il adopte une perspective systémique, interactive alors que la recherche se déroule dans le milieu naturel des personnes ». Après avoir jeté les fondements méthodologiques de notre recherche qualitative, nous allons maintenant présenter les résultats obtenus de l'analyse des entretiens semi-directifs avec les apprenants dans l'ordre des questions posées lors des entrevues.

Les apprenants de notre échantillon déclarent qu'ils ont appris la langue française par les manuels Café Crème (Trevisi et al., 1997) et Reflets (Capelle \& Gidon, 1999). En ce qui concerne le panorama de littérature, y compris la poésie, dans ces manuels, ils mentionnent qu'il y a des extraits de littérature mais rarement de la poésie. L'enquêtée $\mathrm{n}^{\circ}$ 1 qualifie les poèmes dans le manuel Café-Crème de légers : « il n'y a pas de vrais poèmes dans ce manuel, mais des poèmes légers et populaires comme les textes des chansons populaires ». L'enquêté $\mathrm{n}^{\circ} 2$ dit qu'il y a des poèmes dans le manuel Reflets, mais ils sont tellement rares qu'il ne se les rappelle plus.

Pour les apprenants de notre échantillon, la poésie est considérée comme un genre qui appartient plutôt au champ affectif. À ce sujet, quand on a demandé à l'enquêtée $\mathrm{n}^{\circ} 1$ "Quand vous pensez à la poésie, quels sont les premiers mots qui vous viennent à l'esprit?", elle indique: «les sentiments, l'amour, la mort, le chagrin, l'humanité ». 
L'enquêté $\mathrm{n}^{\circ} 2$ considère la poésie comme une expression de "l'état d'âme ». On constate que quand les apprenants évoquent la poésie, des termes affectifs reviennent régulièrement. Ici nous avons bien un trait attribuable à un imaginaire collectif iranien.

Concernant la description de la poésie, les visions des enquêtés sont variées; pour l'enquêtée $\mathrm{n}^{\circ} 1$, l'aspect affectif des poèmes mis à part, l'apprentissage des poèmes est important pour son aspect esthétique. D'après cette enquêtée : «La plus belle image de chaque langue, c'est sa poésie ». Dans cette déclaration, tous les mots mériteraient commentaire: belle, image, langue. D'abord l'adjectif «belle» insiste sur l'aspect esthétique de la poésie, à la suite de quoi la poésie est présentée comme une image de la langue, une représentation de la langue et pas la langue elle-même : dans ce cas, l'image n'est qu'un reflet, mais on peut se demander si cette image entretient un rapport analogique avec ce qu'elle représente ou non. Décrire la poésie comme image de la langue est aussi une manière de matérialiser la poésie qui est avant tout une entité orale, car l'image véhicule un caractère d'objectivité par rapport aux paroles. L'enquêté $\mathrm{n}^{\circ} 2$ dans la description de la poésie, distingue la dimension individuelle de la dimension collective des poèmes, il mentionne que : «La poésie à [s]on avis nous montre d'abord l'état d'âme du poète, et l'image qu'il souhaite donner de son époque». En utilisant le terme " souhaiter " dans son expression, on remarque que même quand il indique la dimension sociale des poèmes, il insiste sur des idéaux du poète pour sa société.

Les enquêtes de notre échantillon soulignent que les textes poétiques dans les cours de français en Iran sont nécessaires. L'enquêtée $n^{\circ} 1$ indiqué que :

C'est très utile, en Iran la poésie est notre identité culturelle, je pense, notre public pense que nos poèmes sont les plus beaux dans le monde et quand les étudiants découvrent les poèmes des autres nations, tout de suite ils vont comparer ces poèmes avec les poèmes iraniens, ça motive les étudiants à parler, à donner leurs avis, c'est utile, en même temps ils vont connaitre aussi la culture de la langue cible.

21 Cette enquêtée prend en compte le rapport à la poésie chez les apprenants iraniens du FLE : pour elle ce rapport est calqué sur l'expérience de la lecture des poèmes persans (le déjà-là), ce qui indique la tendance des étudiants à comparer les poèmes français et persans. Il semble que ce désir influe sur les attentes des apprenants dans la classe de langue. Mais quand on l'interroge sur sa propre expérience de lecture des poèmes dans ses cours de langue, elle dit :

Nous avons eu les poèmes dans nos cours, mais c'est le professeur qui parlait et expliquait, pas les étudiants, nous n'avions pas l'occasion de discuter, on utilisait les poèmes comme les autres textes, c'était un outil pour la compréhension des textes, on travaillait beaucoup sur le vocabulaire, nous ne pouvions pas comparer ou insister sur l'aspect poétique dans la classe.

Dans ces deux propos nous constatons un décalage entre l'attente de l'apprenant et les genres d'activités ou le modèle didactique/ergonomique utilisés dans la classe, à savoir l'éco-poésie (Favriaud, Vinsonneau \& Poletto, 2017) mise en œuvre par l'enseignant dans ces cours.

Dans le discours de l'enquêté $\mathrm{n}^{\circ} 2$ aussi, on trouve l'attente d'un travail plus profond sur les poèmes :

Il n'y a pas assez de temps dans les cours de langue pour travailler sur les poèmes, sauf dans les cours destinés spécifiquement à l'apprentissage de la poésie, cependant dans ces cours non plus, on ne travaille pas vraiment sur les poèmes, mais sur les règles de versification et les figures de style, on étudie les poèmes d'une manière, je dirais, très technique, en plus le cours était trop court. 

qui traitent plus ou moins le même thème, on a l'occasion de mettre l'apprenant face à une multitude de points de vue. Ainsi, dans toutes les langues, nombreux sont des poètes qui ont chanté les paysages familiers, les ravages de la guerre, la beauté de leur ville natale, etc. Ces poèmes peuvent être euphoriques ou dysphoriques, allant du plein au vide, de l'extériorité à l'intériorité :

Le regard est parfois porté par ceux qui saisissent les lieux de l'intérieur, les natifs, comme diraient les Américains (regard endogène). Quelquefois, le regard embrasse le paysage de l'extérieur, lorsqu'on est de passage, lorsqu'on imagine à distance (regard exogène). Il arrive aussi que le regard se joue de la fragile et contestable frontière entre intérieur et extérieur. Il est jeté alors par ceux qui appartiennent conjointement à l'ailleurs et à l'ici, qui vivent en un lieu depuis assez longtemps pour avoir au moins en partie remisé dans leur mémoire leurs origines. C'est le regard allogène de l'exilé et de l'immigré, de celui qui a choisi dans l'euphorie ou subi avec amertume un déplacement. (Westphal, 2005, p. 11)

Outre le modèle fréquent de l'explication du texte, qui est généralement la première phase de l'enseignement qui aborde un texte poétique (évidence qu'on peut remettre en cause dans certains cas), on peut mettre l'accent sur l'aspect communicatif du texte, qui passe par une sorte de circuit tripolaire entre le locuteur premier qui est le poète, le locuteur second qui est l'enseignant pour aboutir au locuteur potentiel qu'est l'apprenant actif. Dans ce cas, il ne s'agit pas seulement des communications ordinaires mais bien de la communication des imaginaires singulier et collectif(s) de ces locuteurs. Prenons ici l'exemple d'un texte de C. Baudelaire, «Le Cygne ", dédié à V. Hugo, où le poète parle de Paris et de sa nostalgie envers cette ville qui change. 

une explication détaillée -, il présente surtout la notion d'exil : nombreux sont les exilés qui apparaissent dans le poème. La dédicace du poème à $V$. Hugo, exilé à Jersey, présente le poème comme un éloge de l'exilé. Le poète flâneur de la capitale fait une référence directe à la réalité parisienne de son époque et parle d'un Paris qui se livre (mais pas toujours de bonne grâce) à la modernisation et à la rénovation. Bien que $\mathrm{C}$. Baudelaire ait souvent été un admirateur de la "modernité ", dans ce poème il a la nostalgie du Paris d'autrefois et face à cette rénovation de la capitale il se sent exilé. Il s'agit ici d'un exil intérieur, de la non-coïncidence entre le monde nouveau et le monde maternel, matriciel. Le poème devient une sorte de tombeau du Paris médiéval; il y aurait une quasihomothétie entre cet exilé-là et le cygne. L'apprenant de langue étrangère se trouve aussi dans un champ de ruine ; pour lui aussi, les constructions nouvelles en langue étrangère pourraient défaire le lien matriciel avec la langue première.

N'oublions pas que la poésie de l'exilé est de grande actualité : bien que le phénomène des migrations de population soit "aussi ancien sans doute que l'humanité elle-même: aventures, expéditions, conquêtes, exodes et colonisations » (Noin, 1995, p. 64), renouant avec l'image antique du nomade, le $\mathrm{XX}^{\mathrm{e}}$ siècle a substitué celle du «migrant misérable en quête d'une terre d'abondance » (Defarges, 1994, p. 14). Dans le poème de C. Baudelaire, le poète se sent exilé dans son propre pays; aujourd'hui encore, il y a des poètes et écrivains, comme A. Camus, qui se sentent en proie à une étrange étrangeté, dans leur propre pays même. Être étranger dans son propre pays, sa propre vie est un topos. Platon et Socrate parlent déjà d'une origine rêvée, hors du monde de la caverne. Cette allégorie de la caverne n'est pas liée directement au manque de l'espace matriciel, mais plutôt à l'espace éthéré du monde des idées, qui a amené au néo-platonisme mystique qui inondera l'Iran, de Sohravardi aux contemporains. Dans tous les cas, nous semble-t-il, le manque est au cœur de la vie, comme il est au cœur de la langue, en raison de la nonadéquation des mots et des choses, et donc au cœur de la poésie. Dans la littérature d'immigration, les manques sont discernables: nous nous trouvons souvent face aux poètes qui, soit ayant été obligés de quitter leur pays, chantent leur nostalgie, soit se trouvant dans un pays étranger, expérimentent une vie d'hybride dans un va-et-vient constant entre culture d'origine et culture d'accueil. Ici pour faire une comparaison entre le regard de C. Baudelaire sur Paris et celui d'un poète iranien ayant la nostalgie de son pays d'origine, nous pouvons prendre l'exemple d'un poème de Sépanlou (1940-2015) qui s'intitule « Naviguer sur Téhéran » (Annexe 2).

Le choix du paysage urbain nous semble pertinent par rapport à nos concepts-clés d'imaginaire singulier, d'imaginaire collectif et d'altérité. C'est un lieu par excellence d'intersubjectivité (Meschonnic, 1982), car dans ces poèmes le rapport entre le poète et le paysage n'est pas le rapport entre sujet et objet, mais bien de l'intersubjectif dans le subjectif. C'est aussi un lieu par excellence de transaction imaginaire, qui rappelle le concept d'objet transitionnel chez D. Winnicott (1975), ainsi que le concept d'objet de réparation de M. Klein commenté par M. Collot (1993, p. 49). Ce «thème » est au cœur de notre problématique sur les fonctions des imaginaires et du déjà-là des apprenants, comme lieu de rêve, dilatation imaginaire de l'énonciateur du poème.

31 En lisant "Naviguer sur Téhéran », on a l'impression que le poète parle d'un autre lieu que « d'ici » où il a toujours vécu. Dans les derniers vers, l'emploi de «Plutôt, j'ai consenti à te voir, Comme vie au loin. » et «subir mon destin » semble être une allusion à quelque chose d'inaccessible dans l'espace, mais en réalité inaccessible dans le temps : le poète parle des 
souffrances que la ville a subies, du passé et du présent, des ennuis de cette ville natale. Des termes comme "absence», "souvenir», "le passé» confié "au soleil» nous montrent que de Téhéran d'autrefois il ne lui reste qu'une image déjà absente d'un passé qui, comme les reflets du soleil, peut lui brûler la mémoire, tout en lui procurant une chaleur agréable. La brûlure de l'esprit reste comme « un discours du dedans ». Le poète est donc un exilé qui, habitant au cœur même de la ville, chante la nostalgie de la ville natale. Si on va plus loin, l'ancien Téhéran est le monde de l'imaginaire singulier, centré autour de l'enfance et de la mère, projection du dedans sur le dehors, qui en devient l'écran, le symbole. C'est également le cas de C. Baudelaire face au Paris d'autrefois. Une comparaison entre les points de vue des deux poètes appartenant à deux époques et à deux cultures différentes, donne l'occasion à l'enseignant de mettre en place des éléments de connivence entre les deux textes poétiques - de la langue maternelle à la langue cible - et, par hypothèse, et entre ceux-ci et l'apprenant tout au long de la séquence.

Une analyse plus profonde et plus littéraire peut mobiliser les concepts de ville-capitale, de ville-femme (mère-amante), les sentiments de désappropriation des lieux et de désappropriation de soi-même. La ville dans ses multiples avatars n'arrive pas à faire retrouver le corps et la langue de la mère, et la langue du poème va tenter d'en trouver un substitut satisfaisant, réparateur. Apprendre une langue étrangère c'est justement tenter de faire ce deuil, de maintenir l'écart entre les deux actants comme possibilité positive de création. En allant d'un texte à l'autre, d'une langue et d'une culture à l'autre, on essaye en quelque sorte de suturer la brisure, la schize. Dans notre lecture, on retrouve également la mère, mais à un autre niveau: on l'introjecte en passant par l'autre.

Cette opération salvatrice peut être favorisée par tel genre d'activités plus que tel autre, par telle " écopoésie » ou milieu d'apprentissage, de création et de réflexivité. Dans le modèle que nous proposons, l'enseignant, comme le psychanalyste, est garant des paroles, de leur circulation, de leur mise en débat réflexif.

Tout d'abord dans une étape de pré-lecture l'enseignant peut demander à l'apprenant de lire le texte dans sa langue maternelle et de le traduire à la suite en français, pour finalement le comparer avec le poème de C.Baudelaire; par exemple, le texte de Sépanlou, cité infra, est la traduction collective des apprenants. Il est à noter que la traduction collective indique un travail de collaboration, une éthique de travail qu'il faut poser comme partie de l'écopoésie. Les apprenants réalisant cette tâche peuvent montrer qu'ils sont capables d'enrichir leurs connaissances grâce à la lecture active-productive des deux textes. En réalité nous pouvons nous référer d'emblée à une «diction expérimentée et musiquée» (Favriaud \& Vinsonneau, 2010, p.50) qui dynamise l'interaction au sein de la classe. Après cette phase de diction qui fait retentir émotionnellement le poème et aide à le mémoriser, nous pouvons nous référer à une lecture interprétative qui « est le miroir où l'on peut se reconnaitre quelles que soient les cultures et les époques. Une évasion qui permet une décentralisation de nous pour aller vers l'autre » (Cervera, 2009, p. 48).

Par ailleurs, dans une classe de FLE et surtout en lisant un texte poétique, les apprenants sont sensibles aux jeux de langage et aux jeux de mots. Ils sont également intéressés à passer d'un sens propre à un sens figuré au moyen de certains tropes tels que l'anaphore ou l'emploi d'un même mot ou groupe de mots au début ou à la fin du vers, ou à la fin ou au début des strophes comme «je pense » dans le poème mentionné de C. Baudelaire ou « 
Comment puis-je subir ton absence » dans ce poème de Sépanlou. Il s'agit du phénomène de paronomase et d'anaphorisation généralisée, relevé par R. Jakobson qui rapproche la poésie de la lallation, de la comptine, de la «lalangue » de J. Lacan (Simonney, 2012), qui est la pré-langue, le corps dans la langue, la tentative de retrouver le même dans l'autre. Cette caractéristique des poèmes peut être efficace pour la réassurance psycho-affective de l'apprenant lors de l'apprentissage d'une langue étrangère.

Le texte poétique, comme tout texte littéraire, mélange la réalité et l'imaginaire et c'est dans le décalage entre les deux que peut se développer la prise de conscience interculturelle tellement prônée dans l'enseignement d'une langue vivante. Lire la poésie est, d'une part, une activité langagière : le poème pousse le lecteur à penser la langue par la voix et l'intègre dans

cet espace formel où l'attention la plus scrupuleuse est portée au langage, où la concentration de (et sur) la langue est la plus vive. C'est donc bien le lieu où s'ajointe de la façon la plus décisive le travail sur la forme et sur le « fond » à savoir les idées et les images. D'où son extrême importance dans l'enseignement, puisqu'elle permet aussi bien de mettre en examen le contemporain que notre parole et notre mémoire. Elle invite les étudiants à travailler indirectement sur eux-mêmes, leur histoire et leur propre capacité articulatoire. (Maulpoix, 2001).

D'autre part, c'est une occasion de vivre l'altérité à travers une fiction et une réalité autre que la sienne. Mais, dans une autre perspective, nous pouvons dire en même temps avec Y. Bonnefoy $(1988$, p. 32) que "la poésie est le dire commun simplement porté à son intensité la plus grande ». Ces thèmes communs dépassent les frontières et les époques et comme nous le dit aussi J.-M. Adam (1991, p. 38): «la poésie, pratique discursive essentielle, a pour fonction de relier socialement aussi bien les différents individus que les différentes époques ».

À première vue, il y a une contradiction entre ces deux perspectives, à savoir vivre l'altérité à travers la poésie et le fond anthropologique commun de la poésie mentionné par ces auteurs. Mais cette contradiction est fertile, car dans ou par la poésie le « radicalement subjectif » touche l'universel et fait comprendre-questionner l'universel, et donc a une fonction sociale de "relation » interhumaine, «transsubjective » selon les termes d'H. Meschonnic (1982) et de S. Martin (2002).

Le plaisir que donne la lecture ou l'écoute des poèmes ci-dessus cités n'est pas seulement dû à la ville décrite, ni à l'intertextualité ou aux évocations mythologiques, mais à un langage où tout fait sens: le signifié des mots comme le matériau sonore, visuel, rythmique de la langue. Et un poète comme Sépanlou, tout en parlant de sa nostalgie, avec un vocabulaire thématique lié au sujet abordé, crée de même une "forme-sens ", pour reprendre le mot composé d'H. Meschonnic, et dans cette perspective c'est la forme, le matériau linguistique qui fait la transaction intersubjective. Sa poésie fait aussi référence à des réalités socioculturelles de son temps, vécues et connues par une grande partie de ses concitoyens. Nous avons dit aussi que dans les deux cas, la ville était une image rêvée meurtrie de soi. Dans une étude comparative entre les deux poèmes, l'enseignant peut également aider l'étudiant à découvrir les divergences tenant à la culture et à l'époque de chaque poète. Nous avons montré aussi de grandes convergences : finalement un imaginaire subjectif assez proche, et un imaginaire collectif, culturel assez différent; tout en sachant que Sépanlou connaissait la poésie européenne et française. 


\section{Conclusion} classe du FLE, la poésie, par sa forme particulière, constitue un moyen pertinent de l'apprentissage du français aux étudiants étrangers, notamment iraniens. Nous avons montré que les apprenants du FLE en Iran prennent plaisir à lire les poèmes en français et manifestent un désir pour des activités d'apprentissage qui insistent plutôt sur la dimension poétique des poèmes que sur les apports technicistes de ces textes. Nous avons vu qu'une comparaison entre deux ou plusieurs poèmes appartenant à la langue maternelle et à la langue cible de l'étudiant peut lui faire vivre une expérience interculturelle et une expérience de respect pour l'autre, ce qui fait cruellement défaut dans certaines sociétés contemporaines. Dans ce dialogue de cultures par le biais de l'enseignement du texte poétique, les divergences d'interprétation sont à percevoir non pas comme des obstacles, mais comme un enrichissement qui permettra aux étudiants de mieux comprendre l'autre. L'enseignant doit aider les apprenants à découvrir la culture française ou francophone dont le texte français est issu et il doit les amener à comprendre les différences des perceptions par rapport à leur milieu, sans négliger les textes issus de leur propre culture, susceptibles d'enrichir cette dernière en retour. Plus précisément nous avons observé que les étudiants veulent une implication plus forte dans la poésie, dans le ressenti de la poésie comme préalable aux activités de culture et langue. Pour ce qui est de la langue, nous n'avons pas observé un refus de la langue, mais le refus d'une stylistique désuète : le relevé des figures de style, les lois de versification quand il est sans enjeu sémantique et émotionnel. L'analyse des entretiens effectués avec les étudiants montre que certaines activités mobilisant un très gros travail de langue, même de réflexivité sur la langue, avaient leur acquiescement, à condition qu'il y ait une forte implication psycho-affective : la traduction, qui peut être, avec la diction et les ateliers d'écriture poétique, l'un des côtés d'un triangle d'activités rénovées.

Pour mieux exploiter des poèmes dans la classe du FLE en Iran sur les plans psychoaffectif, motivationnel, culturel et linguistique, nous proposons une pédagogie qui prend suffisamment en considération le déjà-là des œuvres persanes, l'écopoésie et des différentes tâches et attitudes de l'enseignant.

\section{BIBLIOGRAPHIE}

ADAM, J.-M. (1991). Langue et littérature. Analyses pragmatiques et textuelles. Paris : Hachette.

BONNEFOY, Y. (1988). « La poésie à l'Université ». Le français dans le monde numéro spécial, p. 32-41. BUCHETON, D. (2005). « Présentation générale ». La Lettre de l'AIRDF 36, p. 19-20. En ligne : http:// www.persee.fr/doc/airdf_1776-7784_2005_num_36_1_1644.

CAPELLE, G. \& GIDON, N. (1999). Reflets 1, 2. Paris : Hachette.

Pratiques, $179-180 \mid 2018$ 
CARNUS, M.-F. \& TERRISSE, A. (dirs) (2013). Didactique clinique de l'EPS. Le sujet enseignant en question. Paris : Éd. EP\&S.

CERVERA, R. (2009). « À la recherche d'une didactique littéraire ». Synergie Chine 4, p. 45-52. Collot, M. (1993). « Écriture et réparation dans l'œuvre de Supervielle ». Littérature 90, p. 49-61. En ligne : https://www.persee.fr/doc/litt_0047-4800_1993_num_90_2_2639.

DEFARGES, Ph. M. (1994). Introduction à la géopolitique. Paris : Seuil.

FAVRIAUD, M. \& VINSONNEAU, M. (2010). « Ce que les élèves au cycle 2 vivent et pensent avec la poésie intéresse-t-il la poétique autant que la didactique? ». Le français aujourd'hui 2 (169), p. 49-61. En ligne : https://www.cairn.info/revue-le-francais-aujourd-hui-2010-2-page-49.htm\#.

favriaud, M., vinsonneau, M. \& Poletto, M. (2017). Les Chemins de poésie d'Alep. Poétique et didactique du dire-lire-écrire à l'école primaire. Limoges : Lambert-Lucas.

JORRO, A. (2002). Professionnaliser le métier d'enseignant. Paris : ESF.

LEGENDRE, R. (2005). Dictionnaire actuel de l'éducation. Montréal : Guérin.

MARIN, B. \& CRINON, J. (2017). « Le déjà-là et le presque-là ». Pratiques 173-174. En ligne : http:// journals.openedition.org/pratiques/3364.

MARTIN, S. (2002). «L'historicité radicale du langage ». Acta fabula 3 (2). En ligne : http:// www.fabula.org/revue/document11129.php.

MAUlPoIX, J-M. (2001). « Université \& Poésie ». Zigzag Poésie. En ligne : http://www.maulpoix.net/ universite.html.

MESCHONNIC, H. (1982). Critique du rythme. Anthropologie historique du langage. Lagrasse : Verdier.

MOORE, D. \& PY, B. (2008). «Introduction : discours sur les langues et représentations sociales ». In : Zarate, G., Lévy, D. \& Kramsch, C. (dirs), Précis du plurilinguisme et du pluriculturalisme. Paris : Éd. des Archives contemporaines, p. 271-279.

Moscovici, S. (1984) [1969]. « Préface ». In : Herzlich C. (éd), Santé et maladie. Analyse d'une représentation sociale. Paris : Mouton, p. 7-12.

NOIN, D. (1995). Géographie de la population. Paris : Masson.

RASTIER, F. (1996). «Communication ou transmission ? ». Texto ! 3 (2). En ligne : http://www.revuetexto.net/Inedits/Rastier/Rastier_Transmission.html.

SAVOIE-ZAJC, L. (2004). « La recherche qualitative/interprétative ». In : Karsenti, T. \& Savoie-Zajc, L. (dirs), La recherche en éducation. Étapes et approches. Sherbrooke : CRP, p. 123-150.

SÉPANLOU, M-A. (2009). « qayeq savari dar Tehran » [« Faire du bâteau à Téhéran »]. In : Manzoumeye Tehran [Recueil sur Téhéran]. Téhéran : Nashr-e Ofoq.

SHAYEGAN, D. (2017). L'Âme poétique persane. Paris : A. Michel.

SIMONNEY, D. (2012). «Lalangue en questions ». Essaim 29, p. 7-16. En ligne : https:// www.cairn.info/revue-essaim-2012-2-page-7.htm.

TREVISI, S. et al. (1997). Café crème. Méthode de français. Paris : Hachette.

VERGNAUD, G. (2013). « Carnus Marie-France \& Terrisse André (dir.), Didactique clinique de l'EPS. Le sujet enseignant en question ». Revue française de pédagogie 182, p. 146-147. En ligne : https:// journals.openedition.org/rfp/4043. 
WESTPHAL, B. (2005). L'œeil de la Méditerranée. La Tour d'Aigues : Éd. de l'Aube.

WinNicotT, D.W. (1975) [1971]. Jeu et réalité. Trad. de l'anglais par C. Monod et J.-B. Pontalis. Paris :

Gallimard.

\section{ANNEXES}

\section{Annexe 1}

« Guide d'entretien semi directif avec des étudiants »

I. Parcours individuel de l'informateur

- Par quel manuel méthodologique de FLE vous avez appris le français?

- Comment se dresse le panorama des références à la littérature, y compris la poésie, dans ce manuel?

II. Représentations de la poésie

- Quand vous pensez à la poésie, quels sont les premiers mots qui vous viennent à l'esprit?

- Si je vous demande de décrire la poésie, comment vous la décrirez?

III. Représentations des textes poétiques en classe de langue

- Est-ce que le fait d'utiliser les textes poétiques dans les cours de français en Iran vous semble nécessaire? Pourquoi?

- Avez-vous eu une telle expérience ? Si oui, comment elle était?

IV. Représentations des relations Poésie/Langue et Poésie/Culture

- Comment vous voyez la relation entre la langue et la poésie?

- Comment vous voyez la relation entre la poésie et la culture?

\section{Annexe 2}

« Naviguer sur Téhéran », poème de Sépanlou publié (2006) dans Manzoumeye Téhran ( Recueil sur Téhéran).

\section{Naviguer sur Téhéran}

Tu es l'heure, la lumière, le lit, le silence; Comment puis-je subir ton absence?

Au moins que tu sois endormie* dans tes ennuis.

Tu es le mot, la parole, le baiser, le salut.

Comment puis-je subir ton absence?

Au moins que tu sois morte dans tes lettres.

Tu es le souvenir, la tentation, le discours du dedans;

Comment peux-tu subir mon absence?

\author{
Au moins que je sois mort dans ta mémoire. \\ J'ai révisé les prétextes. \\ J'ai confié le passé au soleil, \\ Je me suis livré à la mort de l'amour. \\ Plutôt, j'ai consenti à te voir \\ Comme vie au loin. \\ Et $j$ 'ai consenti à vivre \\ Courbé sous mon destin.
}

* Dans son poème, Sépanlou s'adresse à Téhéran comme on peut s'adresser à sa bienaimée, c'est pourquoi dans la traduction du poème « tu » est considéré comme féminin. Il y a même un recueil de poèmes consacré à Téhéran et intitulé « La Dame du temps ».

\section{NOTES}

1. Pour une analyse détaillée du lien entre la société iranienne et la poésie voir Shayegan, 2017.

2. Nous empruntons ces notions à M. Favriaud, qui les développe dans la livraison même. 


\section{RÉSUMÉS}

Cet article vise à analyser la place et les fonctions de la poésie française dans l'enseignement du français langue étrangère (FLE) en Iran. Le but est de repérer les rôles et les expériences vécues de l'introduction du texte poétique dans les cours du FLE, tels qu'ils sont perçus par les apprenants du FLE en Iran. À partir des représentations sociales que les apprenants iraniens se forgent de la poésie française, nous présenterons une séquence didactique sur le paysage urbain dans la poésie de C. Baudelaire et de Sépanlou: les deux sont des poètes de ville, Paris pour C. Baudelaire et Téhéran pour Sépanlou. Une comparaison entre les mots et les images utilisés par les deux poètes serait une occasion pour l'apprenant de se plonger dans l'univers imaginaire d'un poète autre que celui de son pays. Les résultats de notre enquête de terrain montrent que la poésie, surtout si on travaille la diction et la production écrite, crée de la passion, de l'attention et de la mémorisation pour la langue seconde, et aussi de la réflexion-réflexivité, si l'enseignant sait accompagner le mouvement de l'imaginaire à la création, et de la création à la réflexivité.

This paper aims at analyzing and discussing the place and functions of French poetry in the teaching of French as a Foreign language in Iran. The purpose of this communication is to identify the roles and lived experiences of using poetic texts in FFL courses, as perceived by the learners of the FFL in Iran. From the social representations that the Iranian learners form of French poetry, we will present a didactic sequence on the urban landscape in the poetry of C. Baudelaire and Sepanlou: the two poets are city poets, Paris for C. Baudelaire and Teheran for Sepanlou. A comparison between the words and the images used by the two poets, will be an occasion for the learner to immerse himself in the imaginary universe of a poet other than that of his country. In all, the results of this study reveal that poetry, especially if we work on diction and written production, creates attention, memorization, passion for the second language, and also reflection-reflexivity, if the teacher knows how to accompany the movement from the imaginary to the creation, and from the creation to the reflexivity.

\section{INDEX}

Mots-clés : poésie, français langue étrangère (FLE), traduction, étude comparative, altérité, compétence interculturelle

Keywords : poetry, FFL, translation, comparative study, otherness, intercultural competence

\section{AUTEURS}

\section{JALEH KAHNAMOUIPOUR}

Université de Téhéran, IR-1417466191, Iran

AKBAR ABDOLLAHI

Université de TéhéranUniversité de Téhéran, IR-1417466191, Iran 